\title{
Gesundheitswirtschaft in Deutschland: Was bringt die Zukunft?
}

\author{
Heinz Lohmann
}

(C) Springer-Verlag 2012

Nicht die Politik ist der Treiber des Umbruchs in der Gesundheitswirtschaft, sondern, wie in anderen Branchen auch, die sich verändernde Situation der „Kunden“. Im Gesundheitsbereich sind das die Patienten, die Krankenversicherungen und die öffentlichen Haushalte. Und da ist die Analyse klar. Seit vielen Jahren steigt die Nachfrage nach Gesundheitsleistungen und gleichzeitig sind die finanziellen Mittel aus dem Sozialtransfer knapp. Das hat einen erheblichen Kostendruck erzeugt. Diese Entwicklung wird auch in Zukunft anhalten und neuerdings durch steigende Qualitätserwartungen ergänzt. Hintergrund dafür ist vor allem die beginnende Patientensouveränität auf Basis der wachsenden Transparenz auch auf dem Gesundheitsmarkt. Das Internet ist dabei ein ganz zentraler Faktor. Immer mehr Menschen nutzen es bei gesundheitlichen Fragen intensiv.

Wenn die Menschen mehr über ihre Gesundheit wissen, sind sie auch bereit, privates Geld für Prävention sowie Diagnostik und Therapie einzusetzen. Deshalb boomt der zweite Gesundheitsmarkt. Dieser Trend ist deutlich zu erkennen. Produkte und Dienstleistungen von Gesundheitsanbietern, die hier aktiv sind, erreichen erhebliche Wachstumsraten. Das gilt inzwischen auch für den Zusatzversicherungsmarkt der Krankenkassen. Brillen- und Zahnersatzversicherungen werden seit einigen Jahren lebhaft nachgefragt. In Zukunft wird es viele weitere Versicherungen geben, da der Umfang der gesetzlichen Krankenversicherungen weiter eingeschränkt werden wird.

Wenn aber auch in Zukunft alle Patienten notwendige Leistungen unabhängig von ihrer individuellen finanziellen Leistungsfähigkeit erhalten sollen, muss die Produktivität der Gesundheitsunternehmen, die auf dem ersten Gesundheitsmarkt agieren, nachhaltig gesteigert werden. Dazu ist

H. Lohmann $(\bowtie)$

LOHMANN konzept, Hamburg, Deutschland die Übertragung von modernen Methoden und Technologien aus anderen Branchen zwingend notwendig. Die digitale Industrialisierung der Medizin und die Schaffung von Gesundheitszentren setzt aber die Gestaltung von strukturierter Behandlung voraus. Die Pflege mit ihrer Nähe zu den Interessen der Patienten ist hervorragend geeignet, ihre Expertise in die bevorstehenden Veränderungsprozesse der Gesundheitsanbieter einzubringen. Sie kann für die Zukunft der Gesundheitswirtschaft insgesamt eine entscheidende Rolle spielen. 